\section{Triple-Ligation Strategy with Advantages Over Directional Cloning}

BioTechniques 21:614-619 (October 1996)

Directional cloning is a convenient means of generating recombinant DNA molecules with a minimal background of undesired clones. By using two restriction enzymes that produce heterologous ends, one can minimize vector self-ligation, thereby reducing the amount of work necessary to identify recombinant clones, especially when lacZ blue-white screening is not possible (1). To fully exploit this benefit of directional cloning, however, the vector must be completely digested with both enzymes. This may not always be feasible, especially when the two restriction sites in the vector are adjacent, because some restriction enzymes are ineffi- cient at cutting near DNA ends. When the two sites are overlapping, directional cloning is impossible. One suggestion that has been made is to do the cloning in two steps, with the first step devoted to adding a sacrificial insert to the original vector, to add effectively more distance between the polylinker sites to be used (2). While this method has intuitive appeal, it requires a preliminary, nondirectional cloning step. Furthermore, a cloning strategy that requires only a single ligation reaction will clearly save time, as long as the products of this reaction remain highly enriched for the desired recombinant. We present here a one-step triple-ligation cloning strategy, which alleviates the drawbacks of simple directional cloning and has added benefits as well, including: $(i)$ the ability to insert DNA fragments with heterologous ends into a given vector in either orientation, irrespective of the order of sites in the vec- tor; (ii) the ability to flank an insert with the same restriction site on both sides; and (iii) the ability to avoid the need for partial digestion in cases where a cloning site is not unique.

A ligation reaction containing three fragments (for example, A-B + B-C + $\mathrm{C}-\mathrm{A}$, where enzymes $\mathrm{A}, \mathrm{B}$ and $\mathrm{C}$ generate mutually incompatible ends) produces the desired recombinant at a lower efficiency than two-piece directional cloning, but because the background of undesired clones is so low, the clones to be screened are highly enriched for the recombinant of interest (1). Therefore, recovering this recombinant in screening steps is quick and easy. In addition, the triple-ligation strategy that we have developed can generate recombinant molecules that cannot be generated by straightforward directional cloning. For example, some cloning applications require recombinant molecules in which the inserted fragment is present in the 


\section{Benchmarks}

opposite orientation from that which the vector polylinker sites allow (for example, when transcribing from a given promoter in the vector). When the fragment to be inserted presents no restriction site alternatives to be used, the common recourse is to render the ends blunt and either: ( $i$ ) to ligate directly to blunt-cut, phosphatased vector; or (ii) to add linkers to the ends, then to ligate to vector digested with the appropriate enzyme and phosphatased. Both of these methods are undesirable due to reduced efficiency, additional handling and the necessity to screen both for the presence of the insert and for the desired insert orientation. The use of linkers also adds expense to the procedure. Compared to the alternatives in this scenario, triple ligation reduces both the number of steps required prior to ligation and the amount of screening to be done after transformation.

Our strategy involves digesting the vector into two fragments, each one having one end digested with a unique restriction site (U) outside of the polylinker and the other end digested with one of the enzymes (X or Y) used to generate the heterologous ends on the insert fragment, as shown in Figure 1A. Because this extra unique site lies at some distance from the polylinker, resolution of completely digested vector bands on a preparative agarose gel is possible, whereas in the case of simple directional cloning, resolving doublycut from singly-cut vector is usually impossible. Additionally, even overlapping polylinker sites can be used because they are digested in separate reactions. Moreover, there are two choices for selecting a pair of vector bands to use in the ligation along with the fragment to be inserted. By selecting the bands representing fragments $\mathrm{X}-\mathrm{U}$ and $\mathrm{U}-\mathrm{Y}$, the equivalent of simple directional cloning is achieved. By selecting the bands representing fragments Y-X-U and U-Y-X, the inverted orientation is achieved. In addition, the inserted fragment is now flanked on both sides by both $\mathrm{X}$ and $\mathrm{Y}$ (and by every polylinker site between them in the original vector), a useful condition for further cloning. When one of the cloning sites (e.g., X) is present elsewhere in the vector as well, partial digestion may be avoided by choosing the appropriate orientation so that the second $X$ site is contained within the vector fragment produced by digestion with the other enzyme (Y).

To demonstrate the technique, we have inserted a 1.6-kb EcoRI-NotI fragment containing an open reading frame (ORF) into the 3-kb vector pBluescript ${ }^{\circledR}$ II KS(+) (Stratagene, La Jolla, CA, USA). Transcription of the ORF proceeds in the EcoRI to NotI direction, and the 1.6-kb fragment contains an asymmetrically situated NsiI site 500 bp from the EcoRI end. The vector contains a unique $A f l$ III site $450 \mathrm{bp}$ from the EcoRI site and does not contain a NsiI site. In separate ligations, the insert was cloned into the vector in the two possible orientations described above and shown in Figure 1B. Note that having both of these recombinant plasmids allows generation of sense and antisense RNA from the same promoter. Note also that the product of the second ligation contains the EcoRINotI portion of the polylinker on both sides of the insert. Thus, any single enzyme between EcoRI and NotI, inclusive, can be used to isolate an insertcontaining cassette from this plasmid.

To generate the same product as in simple directional cloning, the ligation reaction contained, in a 1:1:1 molar ratio, the $1.6-\mathrm{kb}$ insert fragment, the 450 bp EcoRI-AflIII vector fragment and the $2.55-\mathrm{kb}$ Not I-AflIII vector fragment. To generate the inverted insert orientation, the ligation reaction contained, in a $1: 1: 1$ molar ratio, the $1.6-\mathrm{kb}$ insert fragment, the $2.55-\mathrm{kb}$ EcoRI-AflIII vector fragment and the 450-bp NotIAflIII vector fragment. Fragments were isolated by electrophoresing on a $0.8 \%$ agarose gel stained with ethidium bromide, excising bands under longwave ultraviolet (UV) light and purifying with GENECLEAN ${ }^{\circledR}$ II (Bio 101, La Jolla, CA, USA). Ligation was carried out with $1 \mathrm{U}$ T4 ligase (Boehringer Mannheim, Indianapolis, IN, USA) for $16 \mathrm{~h}$ at $12^{\circ} \mathrm{C}$. Ligation reactions were desalted and concentrated for electroporation with $n$-butanol (3) and were electroporated at $2.5 \mathrm{kV}, 200 \Omega$, and 25 $\mu \mathrm{F}$ in a Bio-Rad Gene Pulser ${ }^{\circledR}$ (Hercules, CA, USA). One-tenth of each transformation mixture was plated on LB plates supplemented with $100 \mu \mathrm{g} /$ $\mathrm{mL}$ of ampicillin and $25 \mu \mathrm{g} / \mathrm{mL}$ each of 


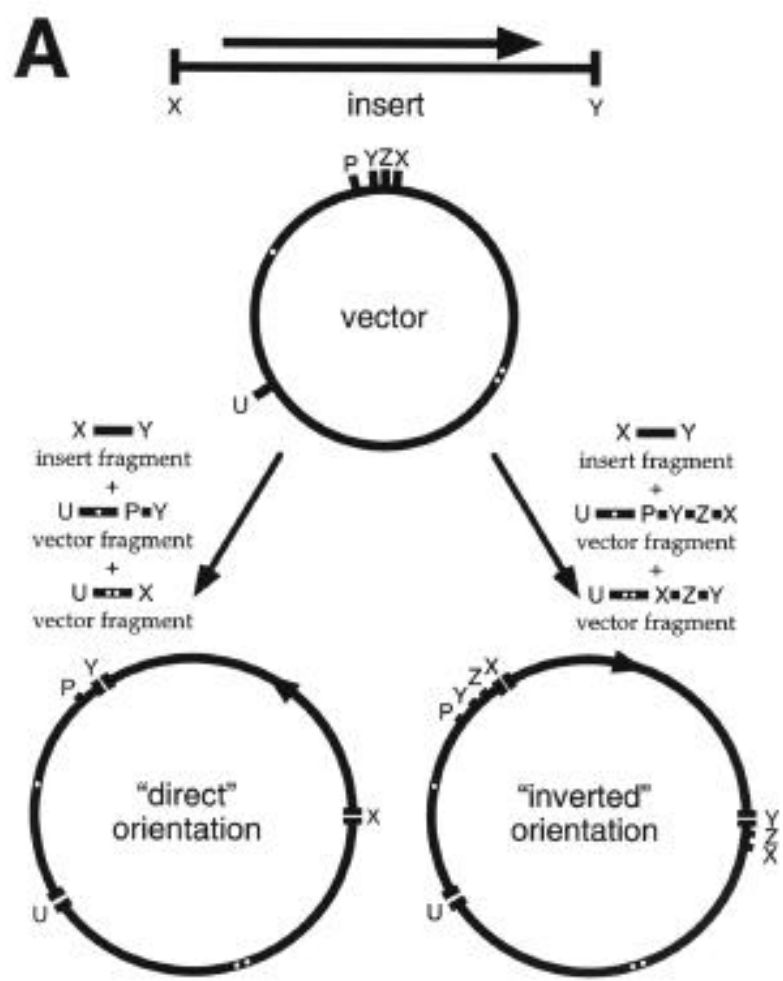

B
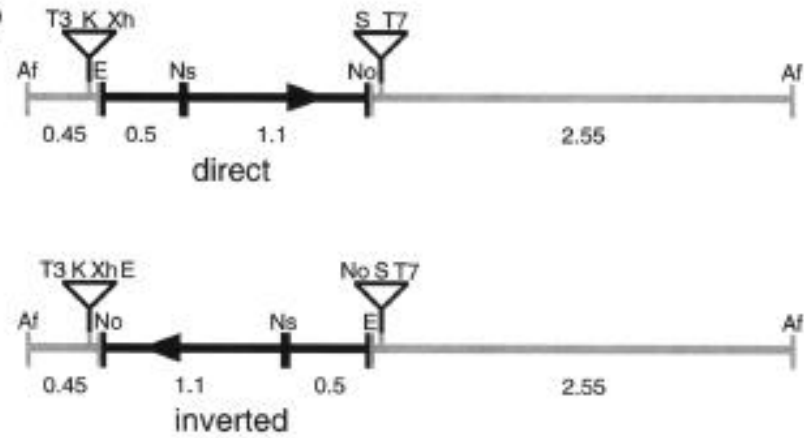

Figure 1. Outline of triple ligation strategy. (A) A DNA fragment with heterologous ends ( $\mathrm{X}$ and $\mathrm{Y}$, arrow shows direction of transcription) is cloned into a vector with $\mathrm{X}$ and $\mathrm{Y}$ sites in its polylinker and a unique site (U) elsewhere. Adjacent to the polylinker lies a promoter $(\mathrm{P})$, from which transcription proceeds in the Y-to-X direction. The vector is digested in one reaction with $\mathrm{X}$ and $\mathrm{U}$ and in a separate reaction with $\mathrm{Y}$ and $\mathrm{U}$. A triple ligation reconstitutes the vector along with the inserted fragment in either direct or inverted orientation relative to the original polylinker sequence. As shown, the desired orientation is achieved by choosing the appropriate restriction fragments from the two vector digestions. Single or double white bullets identify the fragments. Note that in the direct orientation, all polylinker sites between $\mathrm{X}$ and $\mathrm{Y}$ ( $\mathrm{Z}$, for example) are absent, whereas in the inverted orientation, these sites flank the insert on each side. Note also that $\mathrm{P}$ promotes transcription of the anti-sense strand in the direct orientation and the sense strand in the inverted orientation. (B) An ORF-containing fragment with EcoRI (E) and NotI (No) ends is cloned into pBluescript II KS(+) by triple ligation, using the unique AflIII site (Af) in the vector. The arrowhead indicates the direction of transcription. Recombinant plasmids corresponding to direct and inverted orientation are shown, with vector-derived sequences shaded gray. Orientation is evident from the positions of the asymmetrically-situated NsiI site (Ns), the T3 and T7 promoters and the other polylinker sites $(\mathrm{Xh}=\mathrm{XhoI}, \mathrm{K}=K p n \mathrm{I}, \mathrm{S}=\mathrm{SacI})$. The pBluescript II KS $(+)$ vector has five restriction enzyme sites between EcoRI and NotI (not shown in the figure). In the inverted orientation, these sites are present on both sides of the ORF, as are the EcoRI and NotI sites. Each site may be used to derive an ORF-containing cassette from the recombinant plasmid. All distances are in kilobase pairs (kbp). isopropyl- $\beta$-D-thiogalactopyranoside (IPTG) and 5-bromo-4-chloro-3-indolyl- $\beta$-D-galactoside (X-gal). No colonies were blue after $16 \mathrm{~h}$ of incubation at $37^{\circ} \mathrm{C}$ and $8 \mathrm{~h}$ of refrigeration at $4^{\circ} \mathrm{C}$. Individual white colonies were picked for overnight cultures, and plasmid DNA was recovered by alkaline lysis minipreps.

Figure 2 shows diagnostic restriction digests of the two recombinant plasmids. Double digestion with EcoRI and NotI yields the same result for both plasmids: two bands corresponding to insert and vector. Note, however, that the plasmid generated in inverted orientation actually has two EcoRI and two NotI sites and may not be completely digested at all four sites. The direct-orientation plasmid is linearized $(4.6 \mathrm{~kb})$ by EcoRI, whereas the inverted-orientation plasmid yields bands corresponding to insert and vector upon EcoRI digestion. Double digestion of each plasmid with $N s i$ I and XhoI reveals the orientation of the insert in each. The insert's NsiI site is nearer to the vector's XhoI site in direct orientation than it is in inverted orientation. Therefore, double digestion yields bands of sizes $4.1 \mathrm{~kb}$ and $500 \mathrm{bp}$ for the direct-orientation plasmid and bands of sizes $3.5 \mathrm{~kb}$ and $1.1 \mathrm{~kb}$ for the invertedorientation plasmid.

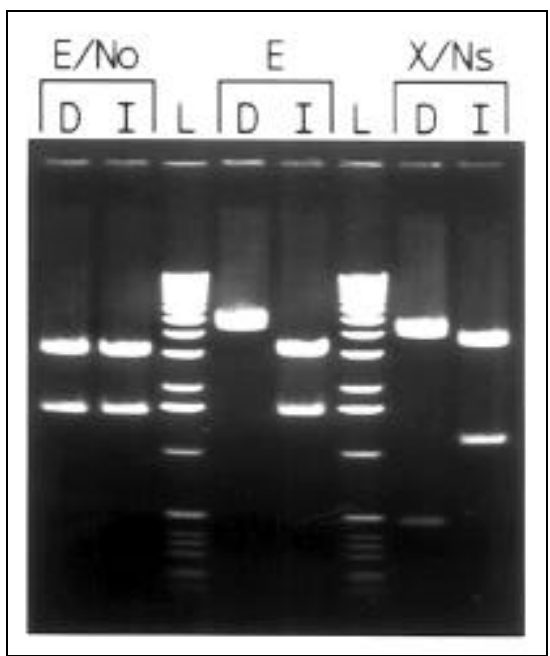

Figure 2. Diagnostic restriction digests of recombinant plasmids. The ORF was cloned both in direct (lanes marked D) and inverted (lanes marked I) orientation into pBluescript II KS(+). Recombinant clones were tested with three restriction digests: EcoRI/NotI (E/No), EcoRI alone (E) and XhoI/NsiI (X/Ns). L = 1-kb ladder (Life Technologies, Gaithersburg, MD, USA). 
The triple-ligation strategy presented here improves upon the concept of directional cloning without forfeiting any of its advantages. The advantages include single-step cloning, high-efficiency generation of the desired recombinant molecule and low background self-ligation of vector. The added benefits of the triple-ligation strategy include easy resolution of vector fragments for gel purification, the ability to invert an insert relative to the orientation of polylinker sites, the ability to flank an insert with the same restriction site on both sides and the ability to use overlapping or otherwise refractory polylinker sites.

Note added in proof: The authors wish to acknowledge the similar treatment of the triple-ligation strategy published recently by Zeng et al. (4).

\section{REFERENCES}

1.Ausubel, F.M. , R. Brent, R.E. Kingston, D. D. Moore, J. A. Smith, J.G. Seidman and K. Struhl (Eds.). 1993. Current Protocols in Molecular Biology, Vol. 1. Greene Publishing Associates and Wiley-Interscience, New York.

2.Buntel, C.J. 1995. Plasmid ligation using a sacrificial insert. BioTechniques 19:351.

3.Thomas, M.R. 1994. Simple, effective cleanup of DNA ligation reactions prior to electro-transformation of $E$. coli. BioTechniques 16:988-990.

4.Zeng, M., B.-R. Huang and J.-N. Lapeyre. 1996. Cloning strategies using a third irrelevant enzyme site (TIES) to overcome certain cloning problems. BioTechniques 20:68-78.

We would like to thank Daniel L. Hartl for the work performed in his laboratory, Lara Brilla for staff support and the other members of the Hartl laboratory. The work was supported by NIH Grants GM33741 and GM07620 and by the Department of Education Training Grant P200A40414. M.L.S. is a Howard Hughes Medical Institute Predoctoral Fellow. Address correspondence to Mark L. Siegal, Department of Organismic and Evolutionary Biology, Harvard University, 16 Divinity Avenue, Cambridge, MA02138USA. Internet: msiegal@ oeb.harvard.edu

Received 27 November 1995; accepted 21 February 1996.

Mark L. Siegal, Dmitri A. Petrov and Daniel De Aguiar Harvard University Cambridge, MA, USA

\section{Useful Properties of Restriction Enzymes That Recognize Interrupted Palindromes}

BioTechniques 21:619-622 (October 1996)

Cloning vectors, which rely on selective markers (1) or indicators in the media (6), are widely used to identify recombinant DNA that carries the intended insert. Nevertheless, situations arise in which these convenient methods are not applicable. Furthermore, if the cloned DNA cannot be distinguished from wild-type DNA by an obvious size difference, the identification of positive clones can become timeconsuming. We describe here an efficient cloning and selection strategy for short synthetic DNA fragments based on the use of restriction enzymes that recognize interrupted palindromes. The approach is conceptually similar to the use of two isocaudomeric enzymes to clone fragments without restoring either restriction site. However, it is generally more applicable and has the additional advantage that essentially $100 \%$ of the positive clones bear the correct insert in the desired orientation. We also describe the construction of a cloning vector that carries recognition sites for three enzymes that recognize interrupted palindromes in its multiple cloning site (MCS).

The approach, as illustrated in Figure 1 , has been recently used to introduce a 26-bp synthetic DNA fragment into the plasmid pCA2c (3), which then encodes an artificial RNA analog of $5 \mathrm{~S}$ rRNA. This expression vector does not carry any selective marker to screen for the desired insertion or the orientation of the cloned fragment.

One nanomole of each synthetic oligonucleotide (5), 5'GTTACATCGTAACATGAGTTTTACGA and 5'GTAACTCGTAAAACTCATGTTACGAT (complementary regions underlined) was annealed to each other in $50 \mu \mathrm{L} 1 \times$ ligase buffer (United States Biochemical, Cleveland, $\mathrm{OH}, \mathrm{USA}$ ) at $90^{\circ} \mathrm{C}$ for 4 min and then cooled slowly down to room temperature. To ligate the resulting DNA fragments into a unique pCA5c recognition site for the endonuclease BstEII (3), 25 fmol of BstEII 